\title{
Application of Pulsed Laser Radiation of the Far Ultraviolet Range for Corneal Crosslinking
}

\section{Igor Kornilovskiy*}

National Medical and Surgical Center Named after NI Pirogov, Ministry of Health of Russia, Moscow

*Corresponding Author: Igor Kornilovskiy, Professor, Doctor of Medical Sciences, Department of Ophthalmology of the Institute of Advanced National Medical and Surgical Center Named after NI Pirogov, Ministry of Health of Russia, Moscow.
Received: February 24, 2021

Published: March 06, 2021

(C) All rights are reserved by Igor

Kornilovskiy.

\section{Abstract}

Purpose: Justify the advisability of using pulsed laser radiation in the far ultraviolet range for corneal crosslinking.

Material and Methods: Experimental (20 pig eyes, 90 rabbit eyes) and clinical (596 photorefractive and phototherapeutic operations) studies on the assessment of corneal crosslinking using ablative and subablative energy densities of an argon-fluorine excimer laser were analyzed. In all cases, stromal saturation with $0.1 \%$ or $0.25 \%$ isotonic riboflavin solution was performed after phototherapeutic ablation of the epithelium. For an objective assessment of the effect of excimer laser crosslinking, the methods of biomechanical testing, light, electron microscopy, computed keratotopography, aberrometry, optical coherence tomography and densitometry were used.

Results: In experiments after phototablation with riboflavin, biomechanical testing revealed an increase in the strength characteristics of the thinned cornea. According to transmission electron microscopy, an increase in the number of cross-links in the collagen structures of the stroma characteristic of cross-linking was observed. In the clinic, OCT and densitometry confirmed the presence of the crosslinking effect in photorefractive and phototherapeutic ablation with riboflavin. Such prophylactic crosslinking was sufficient to compensate for the weakening of the strength properties of the thinned cornea, and did not violate its elastic properties. For therapeutic crosslinking in keratoconus, secondary keratoectasias and other corneal pathology, the radiation energy densities of an argon-fluorine excimer laser below the ablation threshold were applied. With this technology, according to the data on OCT and corneal densitometry, all the classic signs of crosslinking with an increase in optical density, the formation and reverse development of a demarcation line in the stroma were revealed.

Conclusion: Secondary radiation induced by ablative and subablative energy densities of an argon-fluorine excimer laser initiates crosslinking in the riboflavin-saturated corneal stroma. This indicates that the use of pulsed laser radiation of the far ultraviolet range is promising for corneal cross-linking.

Keywords: Pulsed Ultraviolet Laser Radiation; Argon-Fluorine Excimer Laser; Riboflavin; Corneal Crosslinking; Photorefractive Keratectomy; Phototherapeutic Keratectomy; Keratoconus; Secondary Keratoectasias

\section{Abbreviations}

PRK: Photorefractive Keratectomy; TransPRK: Transepithelial Photorefractive Keratectomy; PTK: Phototherapeutic Keratecto- my; FemtoLASIK: Femtolaser in Situ Keratomileusis; CXL: Collagen Cross-Linking; UV: Ultraviolet; UV-A: Ultraviolet-A; UV-B: Ultraviolet-B; D: Diopter; OCT: Optical Coherent Tomography. 


\section{Introduction}

The arsenal of corneal crosslinking technologies is expanding every year. These technologies provide for various methods of saturation of the stroma with riboflavin and its ultraviolet irradiation. For this, as a rule, near ultraviolet with wavelengths of $365 \mathrm{~nm}$ or $370 \mathrm{~nm}$ is used. Irradiation is carried out in continuous or pulsed modes. Our attention was attracted by pulsed laser radiation in the far ultraviolet range, which is widely used in laser photorefractive and phototherapeutic corneal surgery.

\section{Purpose}

Justify the expediency of using pulsed laser radiation of the far ultraviolet range for corneal crosslinking.

\section{Material and Methods}

The work is based on experimental (20 pig eyes. 90 rabbit eyes) and clinical (596 photorefractive and phototherapeutic operations) studies. Particular emphasis was placed on the clinical evaluation of corneal crosslinking using subablative energy densities of an argon-fluorine excimer laser. In all cases, stromal saturation with $0.1 \%$ or $0.25 \%$ isotonic riboflavin solution was performed after phototherapeutic ablation of the epithelium. The operations using laser radiation below the ablation threshold were performed on the Russian excimer laser "MicroScan Visum-500" (Optosystems, Troitsk, Russia). This laser was the first to use an original technical solution that provides a quick transition from ablative to subablative energy densities without performing any additional calibrations. In experimental studies, biomechanical testing of corneal samples, light and electron microscopy were used to assess the state of the cornea after excimer laser crosslinking. In the clinic, the effect of cross-linking was assessed using spectral optical coherence tomography (OCT), keratotopography and corneal densitometry. Corneal OCT was performed using RTVue 100 and RTVue XR100 devices (Optovue, USA). Keratotopographic and densitometric studies were performed using a TMS-5 device (Topcon, Japan). All studies were carried out in compliance with the principles of the Declaration of Helsinki and with the permission of the Ethics Committee of the National Medical and Surgical Center named after NI Pirogov of the Ministry of Health of Russia.

\section{Results}

Comparison of the spectrum of secondary radiation induced by the radiation of an argon-fluorine excimer laser during ablation of epithelium and stroma [1] showed that it completely overlaps all four peaks of maximum absorption by riboflavin (Figure 1). The basis of a new approach to crosslinking during photorefractive surgeries was the rejection of additional ultraviolet radiation and the use of ablation-induced secondary radiation to activate riboflavin.

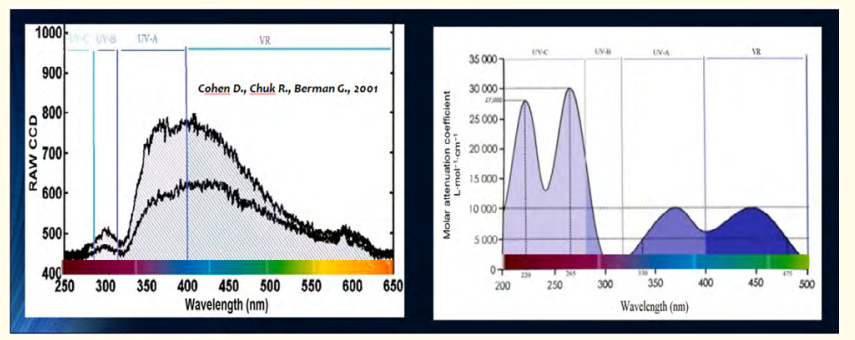

Figure 1: Spectra of induced secondary radiation during corneal ablation (A - epithelium, B - stroma) and spectra of four peaks $(223,268,374$, and $445 \mathrm{~nm})$ of maximum absorption by riboflavin.

Experimental studies on photokeratoablation with stroma saturation with $0.25 \%$ isotonic riboflavin solution revealed the effect of crosslinking and an increase in the strength characteristics of thinned corneal ablation. This was accompanied by a significant increase in tensile strength and maximum tensile strength. Transmission electron microscopy in the stromal layers adjacent to the ablation zone showed an increase in the number of cross-links in collagen fibrils and an increase in the compactness of their packing per unit area (Figure 2). The results of experimental studies were considered in more detail in previously published works [2-5].

The revealed effect of cross-linking was inferior to that with the classical and accelerated technologies of corneal cross-linking. However, it was found to be sufficient to compensate for the weakening of the strong properties of the cornea by thinned ablation. With this prophylactic crosslinking technology, riboflavin saturation of the stroma was carried out for 3-10 minutes, taking into account the degree of ametropia, the volume of removed tissue and corneal pathology. Riboflavin-laden corneal stroma layers protected keratocytes and nerves in deeper stromal structures from ablation-induced secondary radiation (Figure 3). Upon completion of ablation, the effect of crosslinking was initiated in the adjacent stroma layers, which was of a decaying nature. 


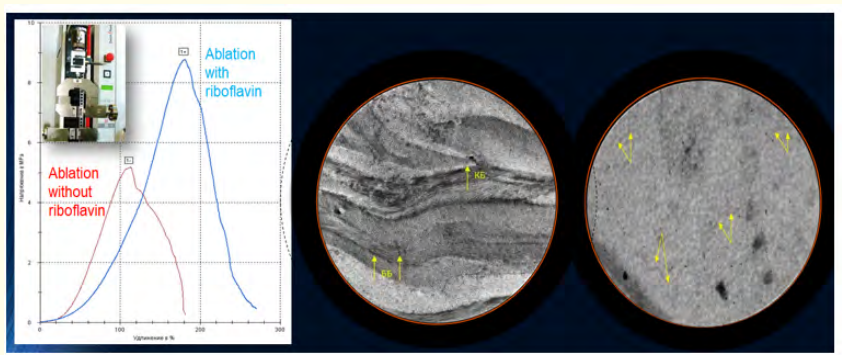

Figure 2: An increase in the strength characteristics of a thinned cornea after phototablation with riboflavin according to biomechanical testing, an increase in the number of interfibrillar cross-links and their compactness in packing according to transmission electron microscopy.

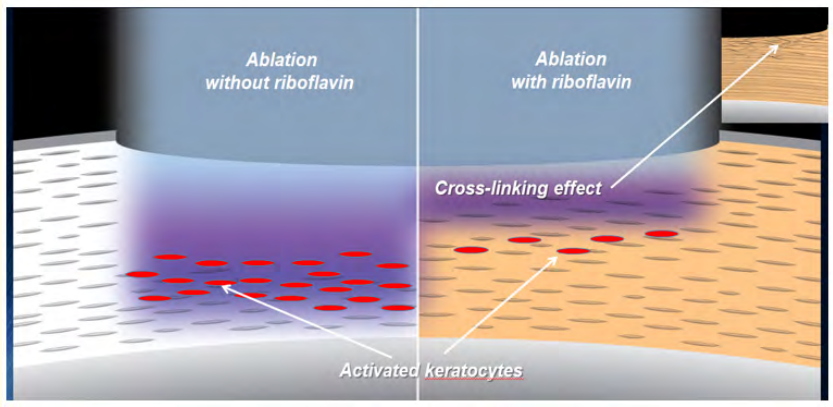

Figure 3: Photokeratoablation without and with saturation of the corneal stroma with riboflavin (diagram).

With this photokeratoablation technology, the quality of the ablative surface improved due to the formation of a Bowman-like membrane structure. On OCT of the cornea, such a membrane structure was detected only when its thickness exceeded $5 \mu \mathrm{m}$, which corresponded to the resolution of the device (Figure 4). OCT and densitometric studies showed an increase in optical density in the stromal layers adjacent to the ablation zone. In isolated cases, after 3-4 weeks, a gentle demarcation line was revealed (Figure 5). With corneal densitometry, an increase in optical density was observed in the stromal layers adjacent to the ablation zone. With prophylactic laser-induced crosslinking, the elastic properties of the cornea were not impaired. Visual and optical-refractive results of prophylactic crosslinking have been reviewed in previously published works $[6,7]$.

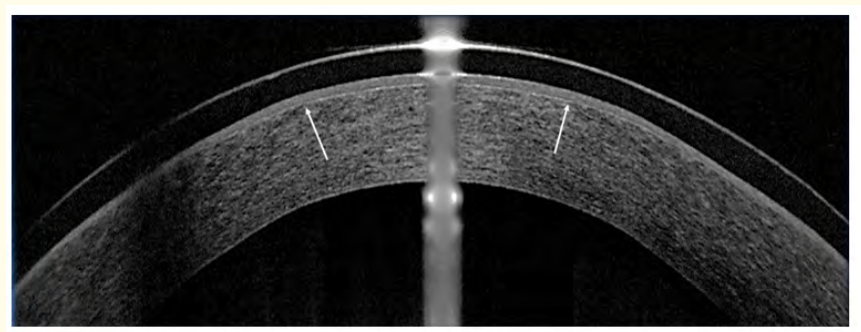

Figure 4: Complete epithelialization under a contact lens 48 hours after TransPRK with riboflavin. In the ablation zone under the epithelium, a Bowman-like membrane structure is determined.

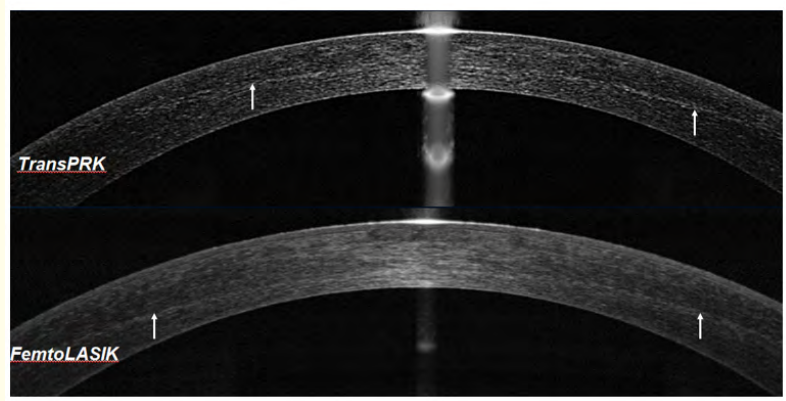

Figure 5: The appearance of a gentle demarcation line a month after the operations TransPRK and FemtoLASIK with riboflavin.

With subclinical signs of keratoconus, the crosslinking effect was enhanced by additional exposure to energy densities below the ablation threshold. A special technology and sub-ablative radiation modes were used to form a Bowman-like membrane structure of greater thickness on the ablative surface. In cases of keratoconus, secondary keratoetasias and other corneal pathologies, a technology for therapeutic corneal crosslinking was developed based on the use of an argon-fluorine excimer laser at energy densities below the ablation threshold. With this technology, the corneal stroma was saturated with $0.1 \%$ or $0.25 \%$ isotonic riboflavin solution. Drip, aerosol and combined saturation techniques were used. For the aerosol technique, an ultrasonic nebulizer with meshtechnology for solution dispersion was used (Figure 6). 


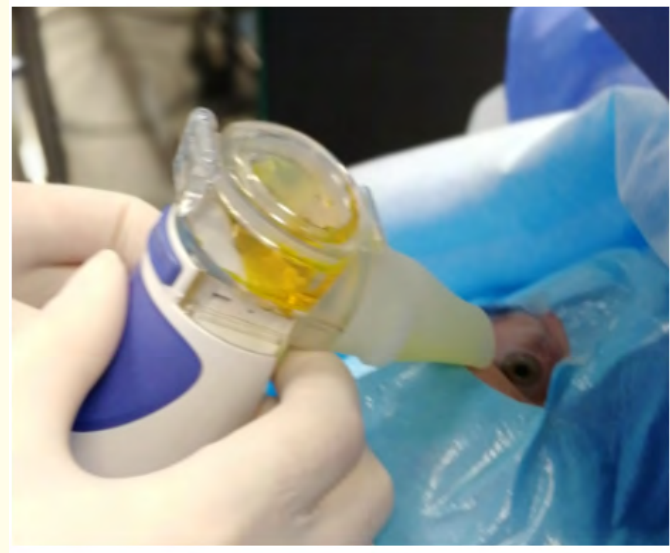

Figure 6: Aerosol saturation of the cornea with riboflavin by means of an ultrasonic nebulizer with mesh-technology of solution dispersion.

Depending on the pathology of the cornea, the saturation time ranged from 5 to 30 minutes. During therapeutic laser-induced crosslinking, according to OCT and densitometry, all the classic signs of traditional crosslinking were revealed (Figure 7). The stabilization or improvement of visual and optical-refractive indices has been traced for 2.5 years and observations are ongoing.
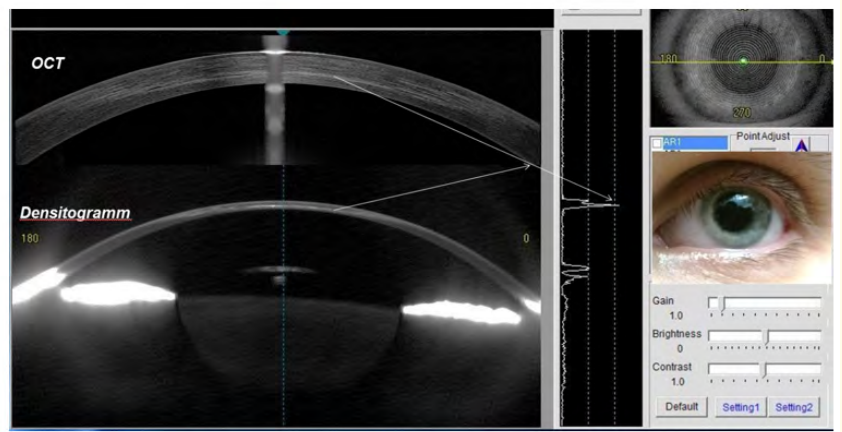

Figure 7: Demarcation line, densitogram and the state of the anterior segment of the eye a month after excimer laser crosslinking for progressive keratocons stage II.

Dynamic OCT and densitometric studies have shown that the timing of the appearance and reversibility of the demarcation line was similar to those with traditional and accelerated technologies of corneal crosslinking [8-10]. In view of the patenting of this technology, specific sub-threshold parameters of laser radiation and the features of scanning the de-epithelialized surface of the cornea are not considered in this article.

\section{Discussion}

The mechanism of laser-induced crosslinking is based on the activation of riboflavin by induced secondary radiation of an argon-fluorine excimer laser with a wavelength of $193 \mathrm{~nm}$. Preliminary theoretical calculations have shown that pulsed laser radiation of the far ultraviolet range of a solid-state laser with a wavelength of $213 \mathrm{~nm}$ and an excimer laser on krypton-chlorine with a wavelength of $222 \mathrm{~nm}$ can have a similar effect. Wavelengths above $222 \mathrm{~nm}$ are mutagenic and carcinogenic and therefore have not found clinical use in corneal surgery. Comparative studies of all of the above wavelengths of far ultraviolet radiation should determine their advantages and disadvantages for prophylactic and therapeutic laser-induced corneal crosslinking.

In laser-induced cross-linking, the absorption of secondary radiation is damped with the maximum effect in the stromal layers immediately adjacent to the ablation zone. This opens up new possibilities for corneal cross-linking with corneal thickness less than $400 \mathrm{~nm}$ and will be presented in our subsequent publications. Moreover, the very fact of using a scanning laser beam with a spot less than $1.0 \mathrm{~mm}$ in diameter made it possible to implement personalized topographically oriented corneal crosslinking. With laser-induced crosslinking, oxygenation in the stroma was less disturbed, which was due to scanning with a laser beam with a smalldiameter spot and a pulsed nature of the radiation. According to recent studies, it is the level of oxygen concentration in the corneal stroma that largely ensures the effectiveness of corneal crosslinking $[11,12]$. Moreover, our observations have shown that the Bunsen-Roscoe law of photochemical interaction is valid for corneal crosslinking in vitro experiments. This law was violated in experiments in vivo, as well as in the clinic with various technologies for corneal crosslinking.

\section{Conclusion}

Secondary radiation induced by the use of ablative and subablative energy densities of an argon-fluorine excimer laser initiates crosslinking in the riboflavin-saturated corneal stroma. This indicates that the use of pulsed laser radiation of the far ultraviolet range is promising for corneal cross-linking. 


\section{Bibliography}

1. Cohen D., et al. "Ablation spectra of human cornea". Journal of Biomedical Optics 6.3 (2001): 339-343.

2. Kornilovskiy IM and Burtsev AA. "Theoretical and experimental substantiation of laser-induced crosslinking in photorefractive corneal surgery". Cataract and Refractive Surgery 15.1 (2015): 20-25.

3. Kornilovskiy IM., et al. "An experimental evaluation of photoprotection by riboflavin in the excimer laser refractive keratectomy". Research Journal of Pharmaceutical, Biological and Chemical Sciences 7.6 (2016): 188-194.

4. Kornilovskiy IM., et al. "Laser-induced corneal cross-linking upon photorefractive ablation with riboflavin". Clinical Ophthalmology 10 (2016): 587-592.

5. Borzenok SA., et al. "The crosslinking effect of photorefractive ablation with riboflavin evaluated with transmission electron microscopy". Pathogenesis 17.2 (2019): 45-50.

6. Kornilovsky IM., et al. "Photoprotection with riboflavin with crosslinking effect in photorefractive corneal ablation". Bulletin of Ophthalmology 132.3 (2016) : 37-42.

7. Kornilovsky IM. "The use of secondary radiation induced by excimer laser ablation for crosslinking in refractive corneal surgery". Cataract and Refractive Surgery 17.3 (2017): 33-40.

8. Kornilovskiy IM. "Optical Coherence Tomography and Densitometry in Assessing the Effect of Corneal Cross-Linking Upon Photorefractive Ablation with Riboflavin". Journal of Eye Study and Treatment 1 (2018): 5-13.

9. Kornilovskiy IM. "Photorefractive Keratectomy with Protection from Ablation-Induced Secondary Radiation and Cross-linking Effect". EC Ophthalmology 10.7 (2019): 563-570.

10. Kornilovskiy IM. "Prophylactic and Therapeutic Laser-Induced Corneal Crosslinking”. EC Ophthalmology 11.12 (2020): 74-82.

11. Lin JT. "Photochemical kinetic modeling for oxygen- enhanced uv-light-activated corneal collagen crosslinking". Ophthalmology Research 7.7 (2017): 1-8, 201.

12. Hafezi N and Hafezi F. "How to choose the best cross-linking procedure in 2016”. European Ophthalmic Review 9.2 (2015): 98-99.

\section{Assets from publication with us}

- Prompt Acknowledgement after receiving the article

- Thorough Double blinded peer review

- Rapid Publication

- Issue of Publication Certificate

- High visibility of your Published work

Website: www.actascientific.com/

Submit Article: www.actascientific.com/submission.php

Email us: editor@actascientific.com

Contact us: +919182824667 